\title{
The retropancreatic fusion fascia acts as a barrier against infiltration by pancreatic carcinoma
}

\author{
HIROHISA KITAGAWA $^{1}$, HIDEHIRO TAJIMA ${ }^{1}$, HISATOSHI NAKAGAWARA ${ }^{1}$, HIRONORI HAYASHI ${ }^{1}$, \\ ISAMU MAKINO $^{1}$, HIROYUKI TAKAMURA ${ }^{1}$, ITASU NINOMIYA ${ }^{1}$, SACHIO FUSHIDA $^{1}$, \\ MASATO KAYAHARA $^{1}$, TETSUO OHTA ${ }^{1}$ and HIROKO IKEDA ${ }^{2}$ \\ Departments of ${ }^{1}$ Gastroenterological Surgery and ${ }^{2}$ Pathology, Division of Cancer Medicine, Graduate School \\ of Medical Science, Kanazawa University, Kanazawa, Ishikawa 920-8641, Japan
}

Received January 7, 2013; Accepted February 6, 2013

DOI: $10.3892 / \mathrm{mco} .2013 .98$

\begin{abstract}
Although pancreatic carcinoma frequently extends posteriorly beyond the pancreatic parenchyma, retroperitoneal organs such as the inferior vena cava (IVC) and the adrenal gland are rarely involved. The fusion fascia lies between the pancreas and these retroperitoneal organs. This study investigated the role of the fusion fascia in the prevention of infiltration of retroperitoneal structures by pancreatic carcinoma. This study was conducted on 140 patients who underwent pancreatic carcinoma resection at our hospital. Retropancreatic infiltration was divided into three grades as follows: Grade 0, carcinoma confined within the pancreatic parenchyma; grade 1, carcinoma infiltrating beyond the parenchyma but within the fusion fascia; and grade 2 , infiltration of retroperitoneal tissues beyond the fusion fascia. Grade 0 was found in $24 \%$, grade 1 in $73 \%$ and grade 2 in $3 \%$ of the cases. There was no significant difference in the prevalence of grade 2 between pancreatoduodenectomy (PD) and distal pancreatectomy (DP). Pancreatic carcinoma infiltrated posteriorly beyond the parenchyma in over $70 \%$ of our cases; however, grade 2 infiltration was a rare finding and tumor invasion was confined within the fusion fascia in almost all the cases. Thus, the fusion fascia may act as a barrier against retroperitoneal tissue infiltration by pancreatic carcinoma.
\end{abstract}

\section{Introduction}

Pancreatic carcinoma frequently infiltrates the anterior and posterior tissues lying beyond the thin adjacent pancreatic parenchyma. The pancreas is fixed posteriorly and the fusion

Correspondence to: Professor Hirohisa Kitagawa, Department of Gastroenterological Surgery, Division of Cancer Medicine, Graduate School of Medical Science, Kanazawa University, 13-1 Takaramachi, Kanazawa, Ishikawa 920-8641, Japan

E-mail: kitagawa@staff.kanazawa-u.ac.jp

Key words: pancreatic carcinoma, retropancreatic infiltration, retropancreatic fusion fascia fascia lies between the pancreatic parenchyma and retroperitoneal organs such as the kidney, adrenal gland and inferior vena cava (IVC). The fusion fascia consists of peritoneal and retroperitoneal elements (1). Pancreatic carcinoma rarely infiltrates the IVC and adrenal gland, which lie beyond this fascia. Therefore, the selection of the retropancreatic dissection plane and concomitant resection of retroperitoneal structures is controversial in pancreatic cancer patients. In the present study, we assessed the role of the fusion fascia in the prevention of the infiltration of tissues located posterior to the pancreas by pancreatic carcinoma.

\section{Materials and methods}

Patient characteristics. This study included 140 patients who underwent pancreatic carcinoma resection at our institution between 1997 and 2011. The age of the patients was 33-80 years (median, 63 years) and the male/female ratio was $84 / 56$. Ninety-one patients underwent pancreatoduodenectomy (PD), 3 underwent total pancreatectomy and 46 underwent distal pancreatectomy (DP). According to the TNM classification of the UICC (2), the tumor was T1, T2, T3 and T4 in 5, 2, 115 and 18 patients, respectively. The disease was stage IA, IB, IIA, IIB, III and IV in 4, 2, 31, 73, 14 and 15 patients, respectively. The histological type was well-, moderately and poorly differentiated in 31, 87 and 21 patients, respectively (Table I). This study was approved by the ethics committee of Kanazawa University. Informed consent was obtained from all patients.

Grading. Resected specimens were cut into 5-mm stepwise tissue blocks following fixation with $10 \%$ neutral buffered formaldehyde solution. Then, consecutive $5-\mu \mathrm{m}$ serial sections were cut from the blocks. PD specimens were cut perpendicular to the body axis, whereas DP specimens were cut perpendicular to their own longitudinal axis. Retropancreatic infiltration was graded as follows: Grade 0, carcinoma confined within the pancreatic parenchyma; grade 1 , infiltration beyond the parenchyma but within the fusion fascia (including tumors abutting the fascia) (Figs. 1 and 2); and grade 2, infiltration of retroperitoneal tissues beyond the fusion fascia (Figs. 3 and 4). 


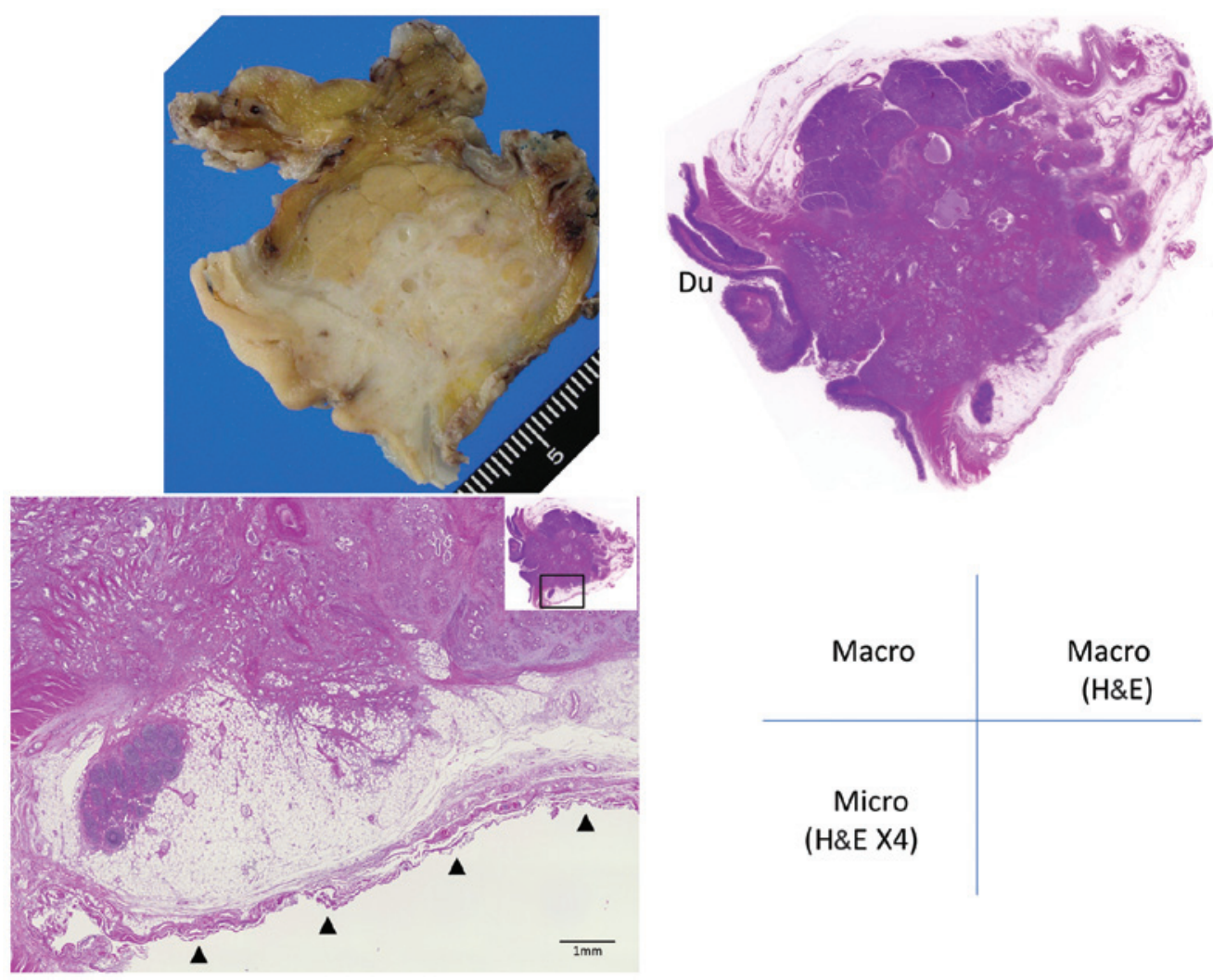

Figure 1. Grade 1 retropancreatic infiltration by pancreatic head carcinoma. The specimen was cut perpendicular to the body axis. The tumor has infiltrated beyond the parenchyma, but remains within the fusion fascia. Arrowheads indicate the fusion fascia. Du, duodenum; Macro, macroscopic view of the resected specimen; Macro H\&E, macroscopic specimen stained with hematoxylin and eosin; Micro (H\&E x4), microscopic section stained with H\&E, under magnification x4.

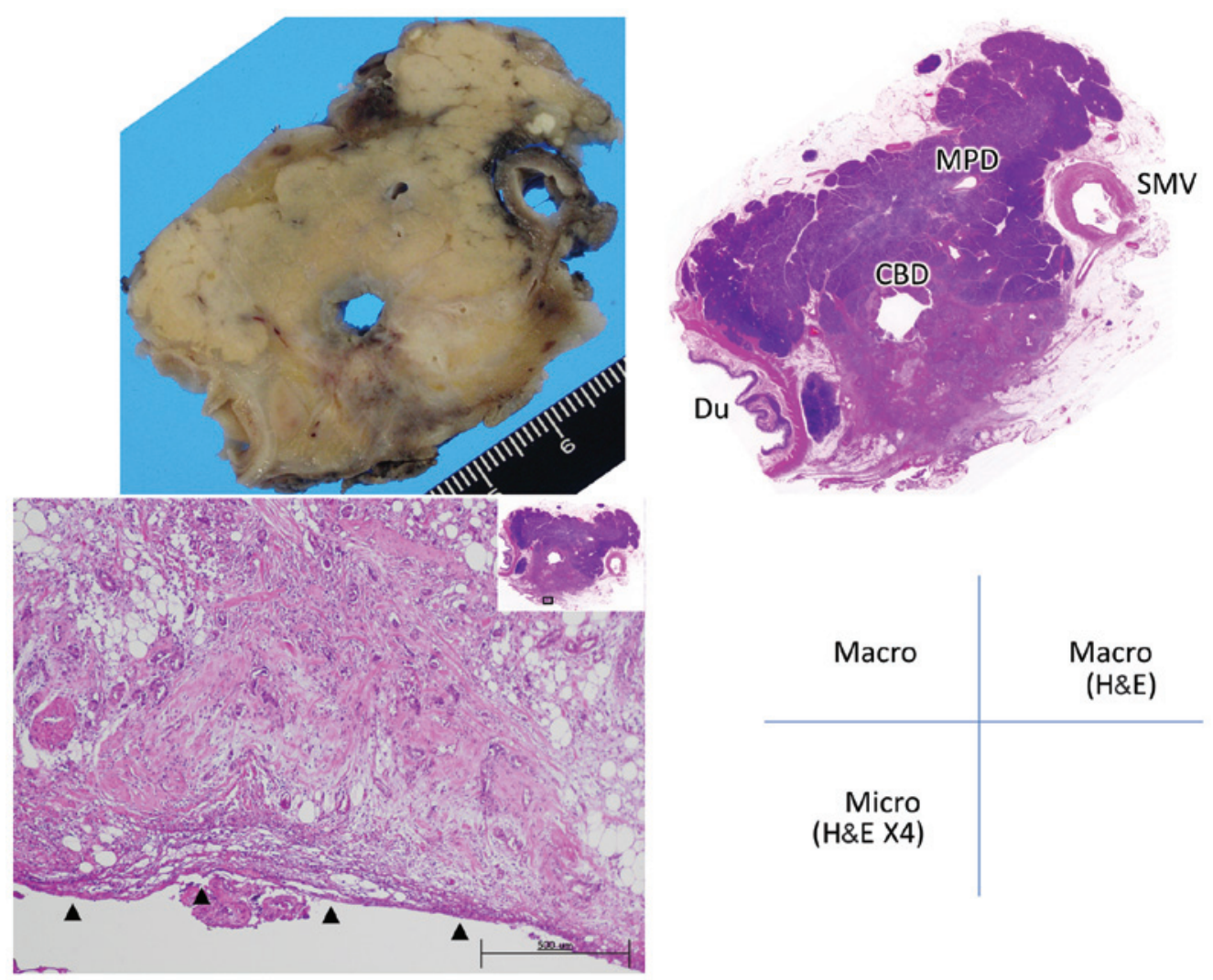

Figure 2. Grade 1 retropancreatic infiltration by pancreatic head carcinoma. The specimen was cut perpendicular to the body axis. The tumor has infiltrated beyond the parenchyma and is adjacent to the fusion fascia. Arrowheads indicate the fusion fascia. Du, duodenum; CBD, common bile duct; MPD, main pancreatic duct; SMV, superior mesenteric vein. Macro, macroscopic view of the resected specimen; Macro H\&E, macroscopic specimen stained with hematoxylin and eosin; Micro (H\&E x4), microscopic section stained with $\mathrm{H} \& \mathrm{E}$, under magnification $\mathrm{x} 4$. 
Table I. Patient baseline characteristics.

\begin{tabular}{lc}
\hline Age in years (range) & $63.2(33-80)$ \\
Gender & \\
Male & 84 \\
Female & 56 \\
Surgical procedure & \\
PD & 91 \\
TP & 3 \\
DP & 46 \\
TNM classification & \\
T1 & 5 \\
T2 & 2 \\
T3 & 115 \\
T4 & 18 \\
Stage & \\
IA & 4 \\
IB & 2 \\
IIA & 32 \\
IIB & 73 \\
III & 14 \\
IV & 15 \\
Histological differentiation & \\
High & \\
Moderate & 31 \\
Poor & 87 \\
\hline
\end{tabular}

PD, pancreatoduodenectomy; TP, total pancreatectomy; DP, distal pancreatectomy.

\section{Results}

The extent of retropancreatic infiltration was grade 0 in $24 \%$ and grade 1 in $73 \%$ of the patients. Grade 2 was observed in merely $3 \%$ of the patients. There was no significant difference in grade 2 infiltration between patients undergoing PD and those undergoing DP (Table II).

Grade 2 infiltration was observed in a total of 4 patients, 2 of which had undergone PD and the other 2 DP. In those patients, the tumor had infiltrated the IVC, adrenal gland and retropancreatic adipose tissue located posterior to the fusion fascia (Table III). Combined resection of the involved tissues was performed in all 4 patients.

\section{Discussion}

Pancreatic carcinoma has the worst prognosis among all gastrointestinal cancers, with a frequency of local recurrence and liver metastases (3-5). Retropancreatic infiltration beyond the parenchyma is common; however, involvement of retroperitoneal organs is rare. Thus, the optimum retropancreatic dissection plane and the need for concomitant resection of retroperitoneal organs such as the adrenal gland, kidney or IVC, remains a controversial subject (6).

During embryological development, the retroperitoneal organs are covered by the anterior renal fascia and the pancreas is covered by the proper peritoneum. The pancreas becomes fixed posteriorly along with rotation of the mesentery during the 6th-12th weeks of gestation (7), after which time the proper pancreatic peritoneum and the anterior renal fascia

Table II. Grade of infiltration by pancreatic carcinoma.

\begin{tabular}{lcccc}
\hline Surgical procedure & No. of patients & Grade 0, n $(\%)$ & Grade 1, n $(\%)$ & Grade 2, n $(\%)$ \\
\hline PD & 91 & $21(23)$ & $68(75)$ & $2(2)$ \\
TP & 3 & 0 & $3(100)$ & 0 \\
DP & 46 & $13(28)$ & $31(68)$ & $2(4)$ \\
Total & 140 & $34(24)$ & $102(73)$ & $4(3)$
\end{tabular}

Retropancreatic infiltration was classified into 3 grades. Grade 0 (carcinoma confined within the parenchyma) was found in 22,28 and $24 \%$ of pancreatoduodenectomy (PD), distal pancreatectomy (DP) and total patients, respectively. Grade 1 (infiltration beyond the parenchyma but within the fusion fascia) was found in 76,68 and 73\% of PD, DP and total patients, respectively. Grade 2 (infiltration of retroperitoneal tissues beyond the fusion fascia) was found in 2,4 and $3 \%$ of PD, DP and total patients, respectively. TP, total pancreatectomy.

Table III. Details of grade 2 cases.

\begin{tabular}{|c|c|c|c|c|c|c|c|c|}
\hline Case no. & $\begin{array}{l}\text { Surgical } \\
\text { procedure }\end{array}$ & $\mathrm{T}$ & $\mathrm{N}$ & M & Stage & $\begin{array}{l}\text { Histological } \\
\text { type }\end{array}$ & $\begin{array}{l}\text { Tumor size } \\
(\max \mathrm{mm})\end{array}$ & $\begin{array}{c}\text { Extrapancreatic } \\
\text { involvement }\end{array}$ \\
\hline 1 & $\mathrm{PD}$ & 3 & 1 & 0 & IIB & Por & 37 & IVC \\
\hline 2 & $\mathrm{PD}$ & 3 & 1 & 0 & IIB & Mod & 38 & IVC \\
\hline 3 & DP & 3 & 3 & 1 & IV & Mod & 40 & Adrenal gland \\
\hline 4 & DP & 3 & 1 & 0 & IIB & Por & & Adipose tissue \\
\hline
\end{tabular}

Two patients underwent pancreatoduodenectomy and 2 received distal pancreatectomy. The involved retroperitoneal organs were the inferior vena cava (IVC), adrenal gland and retroperitoneal adipose tissue. PD, pancreatoduodenectomy; DP, distal pancreatectomy; Por, poorly differentiated; Mod, moderately differentiated. 

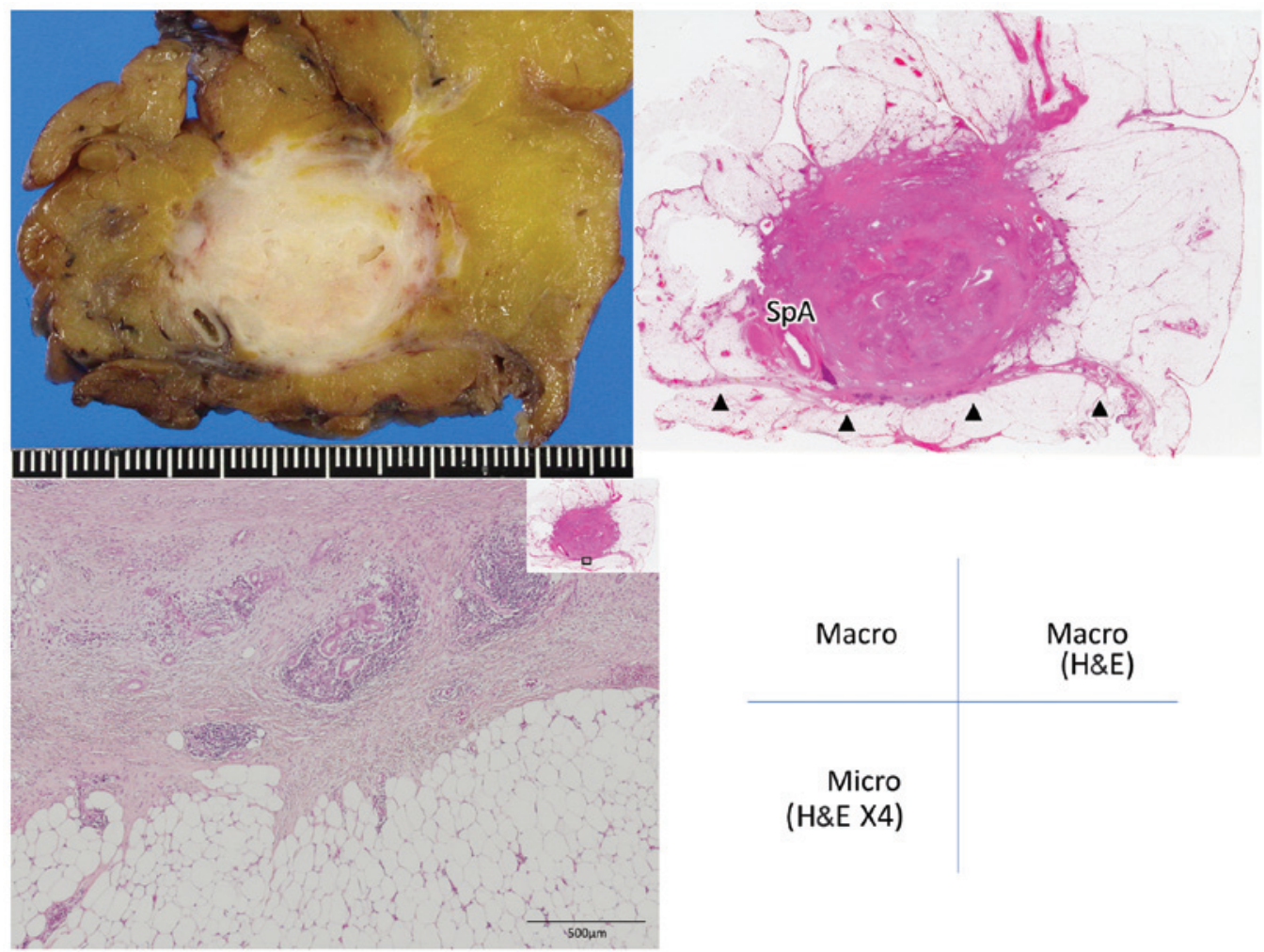

Figure 3. Grade 2 retropancreatic infiltration by carcinoma of the pancreatic body. The specimen was cut perpendicular to the longitudinal axis of the pancreas. The tumor has infiltrated the retroperitoneal adipose tissue beyond the fusion fascia and is accompanied by a desmoplastic reaction. Arrowheads indicate the fusion fascia. SpA, splenic artery; Macro, macroscopic view of the resected specimen; Macro H\&E, macroscopic specimen stained with hematoxylin and eosin; Micro (H\&E x4), microscopic section stained with H\&E, under magnification x4.
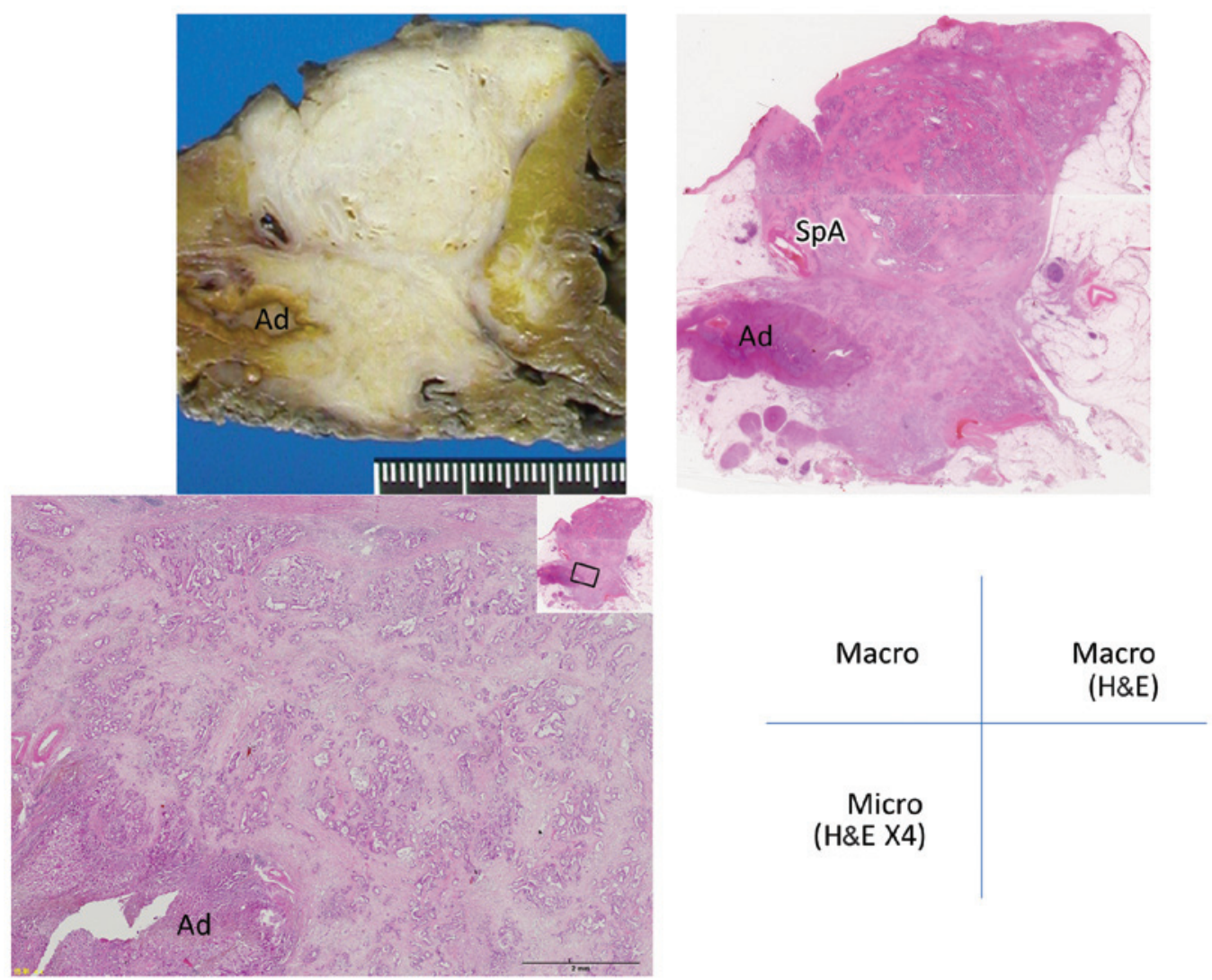

Figure 4. Grade 2 retropancreatic infiltration by carcinoma of the pancreatic body. The specimen was cut perpendicular to the longitudinal axis of the pancreas. The tumor has invaded the adrenal gland beyond the fusion fascia. SpA, splenic artery; Ad, adrenal gland; Macro, macroscopic view of the resected specimen; Macro H\&E, macroscopic specimen stained with hematoxylin and eosin; Micro (H\&E x4), microscopic section stained with H\&E, under magnification x4. 
fuse. This fused membrane plays the role of the retroperitoneal fascia as well as of the proper pancreatic peritoneum.

In the present study, we classified retropancreatic infiltration into 3 grades and identified tumor infiltration beyond the posterior border of the pancreatic parenchyma in $>70 \%$ of our cases. By contrast, infiltration beyond the fusion fascia (grade 2) was a rare finding (3\%). Tumor infiltration was confined to the anterior side of the fusion fascia in the majority of cases, even if the carcinoma was identified as adjacent to the IVC or adrenal gland on preoperative imaging. Thus, the fusion fascia may act as a barrier against infiltration by pancreatic carcinoma.

Kocher's maneuver is usually performed during PD. Following this traditional mobilization procedure, the fusion fascia may be identified as a thin and translucent fibrous membrane covering the surface of the IVC. However, the pancreatic head is resected without the fusion fascia by the traditional Kocher's maneuver. It is not customary to intentionally select this dissection plane, since the fusion fascia is too thin to identify, similar to the peritoneum of the abdominal wall. However, dissection including the fusion fascia is recommended, since this fascia acts as the proper peritoneum of the pancreas and delineates the retroperitoneum.

A dissection plane including the fusion fascia is readily identified anterior to the IVC or the left renal vein. Following exposure of the adventitia of these veins, this plane may be precisely identified.

A microscopically negative resection margin (R0) is the most important prognostic factor for pancreatic carcinoma; however, the posterior margin has little impact on the prognosis compared to the mesopancreatic margin following PD for pancreatic ductal adenocarcinoma (8). Even in the case of a very short distance between the retropancreatic margin and the leading edge of the tumor, an R0 may be obtained by resection including the fusion fascia. Therefore, resection including the fusion fascia is recommended at the posterior margin of the pancreas, since tumor infiltration is confined to the anterior surface of the fusion fascia in the majority of cases.

In conclusion, the fusion fascia lies between the pancreas and the retroperitoneal organs and provides protection against retropancreatic infiltration of pancreatic carcinoma. Pancreatic carcinoma should be resected en bloc with the retropancreatic fusion fascia when surgical resection is performed with curative intent.

\section{References}

1. Perlemuter L and Waligora J: Cahiers d'Anatomie Vol. 2. 3rd edition. Masson et Cie, Paris, 1975.

2. Unio Internationalis Contra Cancrum(UICC):TNMClassification of Malignant Tumours. 7th edition. Wiley-Blackwell, New York, 2010.

3. Kayahara M, Nagakawa T, Ueno K, et al: An evaluation of radical resection for pancreatic cancer based on the mode of recurrence as determined by autopsy and diagnostic imaging. Cancer 72: 2118-2123, 1993.

4. Kayahara M, Nagakawa T, Ueno K, et al: Surgical strategy for carcinoma of the pancreas head area based on clinicopathologic analysis of nodal involvement and plexus invasion. Surgery 117: 616-623, 1995.

5. Nagakawa T, Ohta T, Kayahara M, et al: Clinicopathological evaluation of long-term survivors treated for cancer of the head of pancreas. Hepatogastroenterology 45: 1865-1869, 1998.

6. Strasberg SM, Linehan DC and Hawkins WG: Radical antegrade modular pancreatosplenectomy procedure for adenocarcinoma of the body and tail of the pancreas: ability to obtain negative tangential margins. J Am Coll Surg 204: 244-249, 2007.

7. Moore KL and Persaud TVN: The Developing Human: Clinically Oriented Embryology. 8th edition. Saunders, Philadelphia, 2008.

8. Jamieson NB, Foulis AK, Oien KA, et al: Positive mobilization margins alone do not influence survival following pancreaticoduodenectomy for pancreatic ductal adenocarcinoma. Ann Surg 251: 1003-1010, 2010. 